\title{
Análisis de la calidad de los servicios educativos en Latinoamérica
}

\author{
Deciderio Francisco Mori Apuela \\ deci24@ hotmail.es \\ https://orcid.org/0000-0003-4147-8142
}

Universidad César Vallejo.

Gabriela del Pilar Palomino Alvarado

dpalominoal@ucvvirtual.edu.pe

https://orcid.org/0000-0003-0851-4375

Universidad César Vallejo.

\section{RESUMEN}

La investigación tuvo como objetivo identificar, examinar sistemáticamente la mejor evidencia científica disponible sobre la calidad de los servicios educativos en Latinoamérica, disponible en la base de datos EBSCO, REDALYC, ProQuest, de forma gratuita en inglés y español de los últimos 5 años. Método: Se ingresó a la base de datos EBSCO, REDALYC, ProQuest, realizándose la búsqueda del término "calidad de los servicios educativos", descartándose artículos que no sean arbitradas y académicas, que hayan sido publicados en 2016 o antes, y que no estén disponibles para su descarga gratuita desde EBSCO, REDALYC, ProQuest. Resultados: Tras la búsqueda de artículos relacionados con los conceptos de calidad de servicios educativos se distinguieron que la mayoría de las dimensiones de calidad inherentes al servicio educativo prestado estuvieron por encima del nivel esperado y que su rendimiento fue bueno, ya que mejoran de un periodo a otro. Conclusión: De los resultados obtenidos, se puede evidenciar que existe una buena percepción por parte de los estudiantes y/o usuarios acerca de la calidad de los servicios educativos. El modelo más utilizado para medir la calidad de los servicios educativos es SERVQUAL.

Palabras claves: calidad de los servicios; calidad de los servicios educativos; medición de la calidad de los servicios educativos. 


\title{
Analysis of the quality of educational services in latin America
}

\begin{abstract}
The objective of the research was to identify and systematically examine the best available scientific evidence on educational services in Latin America, available in the EBSCO, REDALYC, ProQuest database, free of charge in English and Spanish for the last 5 years. Method: The EBSCO, REDALYC, ProQuest database was entered, searching for the term "educational services", discarding articles that are not refereed and academic, that have been published in 2016 or before, and that are not available for review. free download from EBSCO, REDALYC, ProQuest. Results: After searching for articles related to the concepts of quality of educational services, it was distinguished that most of the quality dimensions inherent to the educational service provided were above the expected level and that their performance was good, since they improve over a period of time. to another Discussion and Conclusion: From the results obtained, it can be seen that there is a good perception by students and / or users about the quality of educational services.
\end{abstract}

Keywords: educational; services; education.

Artículo recibido: 05 octubre. 2021 Aceptado para publicación: 02 noviembre 2021 Correspondencia: deci24@ hotmail.es Conflictos de Interés: Ninguna que declarar 


\section{INTRODUCCIÓN}

Hoy las instituciones educativas juegan un rol importante en la sociedad, toda vez que se constituyen en uno de los entes de formación y transformación social más importante en cualquier contexto. Desde esta perspectiva los centros de atención del servicio en una institución educativa buscan satisfacer a los usuarios y el contexto en donde usualmente intervienen. Las nuevas realidades de estas instituciones hacen necesario el poder definir y medir las dimensiones de calidad de un centro de atención del servicio. sin embargo, los usuarios son cada día más exigentes, lo que requiere no solo poder definir y medir estas dimensiones de calidad del servicio desde diferentes perspectivas. (Fontalvo, et al. 2020)

La evolución de la forma como las empresas se relacionan con sus clientes va de la mano con las nuevas tecnologías de la información, hace necesario enfatizar en la gestión del factor servicios como un aspecto estratégico para el desarrollo de las organizaciones, considerando que en un mundo de negocios tan competitivo como el actual la diferencia puede estar en una correcta interpretación de las inconformidades de los clientes y la efectividad para traducir dichas inconformidades en acciones de mejoramiento. (Delahozdominguez, et al. 2020)

Los centros educativos, para su funcionamiento, cuentan con docentes, que cumplen la función de autoridad y docentes de aula a la vez, y los demás de enseñanza y tutorías, también encontramos al personal administrativo que cumplen función de oficinistas y recepción a los usuarios y otros que cumplen la función de mantenimiento reparación y atención en los ambientes, laboratorios, aulas y talleres. Cada uno de los miembros de esta organización llamada Institución Educativa se integra para trabajar con sus propias culturas, formas de convivencia, actividades, habilidades y actitudes para su desarrollo de sus propias labores destinadas a atender los requerimientos de los usuarios. (Palacios, Damián, \& Damián, 2020)

Las instituciones de educación deben considerar como política ofrecer servicios de calidad que se reflejen en la satisfacción de los usuarios para captar a más estudiantes y mejorar su eficiencia terminal. La calidad es un factor que distingue a una universidad y le proporciona una ventaja frente a otras instituciones similares, lo cual determina su crecimiento en medio de una fuerte competencia. Debido a esto, se puede afirmar que la educación superior debe ser reconocida como una industria de servicios, la cual se 
encarga de satisfacer las necesidades y expectativas de sus estudiantes o clientes. (Martínez-Luis, et al. 2020)

La satisfacción del cliente es un tema de gran importancia para toda organización que desee lograr una ventaja competitiva, ya que los clientes satisfechos serán fieles a la empresa. Las instituciones educativas no son ajenas a esta realidad. En el contexto empresarial, existen diferentes instrumentos para medir satisfacción de clientes, algunos de uso mundial, que se han adaptado al contexto educativo, como las escalas SERVQUAL y SERVPERF. Existen instrumentos diseñados específicamente para medir la satisfacción de usuarios de instituciones de educación enfocados sólo en universidades públicas y privadas, donde el entrevistado es el usuario directo (estudiante) (Montero-Mora \& Cantón-Croda, 2020)

\section{Calidad de los servicios}

La calidad del servicio es un concepto que ha acogido un gran esfuerzo de investigación en el sector educativo a nivel internacional y local y ha sido empleado para mejorar el servicio desde un punto de vista objetivo y subjetivo como resultado de la interacción, o del encuentro, del cliente con el servicio. (Alonso Dos Santos, 2016)

Por su parte, (Araya-castillo, et al. 2016) La calidad de servicio es vista por las instituciones como una decisión estratégica para satisfacer las necesidades de sus clientes y lograr posiciones competitivas en los mercados.

Asimismo, (Turpo \& Jaimes, 2017), en la última década, la calidad se ha convertido en un concepto citado por las principales instituciones públicas y de servicios, se ha convertido en una meta buscada de una manera completa, ya que se ha considerado de forma común que lo que tiene "calidad" satisface las expectativas del cliente, la calidad en general abarca todas las cualidades de un producto o un servicio, cuando sus características, tangibles e intangibles satisfacen las necesidades del usuario.

También es aquella actividad que relaciona a la empresa con el cliente, a fin que este quede satisfecho con dicha actividad. Actividades orientadas a una tarea, que no sea la venta proactiva, que incluyen interacciones con los clientes en persona, por medio de telecomunicaciones o por correo. Esta función se debe de diseñar, desempeñar y comunicar teniendo en mente dos objetivos: la satisfacción del cliente y la eficiencia operacional. (Sotelo \& Figueroa, 2017) 
La calidad de servicio se define como la diferencia entre el servicio experimentado y el esperado por los usuarios, también puede ser conceptualizado como la excelencia del servicio brindado. (Regina Ferreira \& De la Cruz, 2019)

Desde la óptica exclusiva de calidad en el servicio se presentan diferencias sustanciales dada la necesidad del proceso de medición entendido este como el conjunto de actividades que busca responder las necesidades de un cliente cuyas características son la intangibilidad, inseparabilidad, heterogeneidad y carácter perecedero, las diferencias esenciales entre evaluar la calidad del servicio y la calidad de un producto; a diferencia de la calidad en los productos, en el que la calidad puede ser medida objetivamente a través de indicadores tales como número de defectos; la calidad en el servicio es algo fugaz que puede ser difícil de medir. (Contrera, et al. 2019)

\section{Calidad de los servicios educativos}

Por su parte, (Arciniegas \& Mejías, 2017), afirma que los servicios educativos poseen características propias de la naturaleza del mismo, y la satisfacción del cliente final, como medición final, terminará siendo la obtención de un servicio que abarca las especificaciones requeridas por el cliente, a pesar de las características anteriormente descritas.

Para (Palacios, et al. 2020), la calidad de servicio educativo es definir sobre un nivel en cuanto al servicio que brinda una institución educativa, pero en este sentido es complejo definirlo, ya que cada institución tiene características únicas, por ende, el servicio que brindan también va ser diferente.

El contexto tiene que ver con aspectos sociales que condicionan la operación y desempeño del sistema educativo. Los insumos y recursos están relacionados con la operación de las escuelas y las aulas (alumnos, docentes, directores y escuelas), se incluyen los recursos financieros destinados a la operación del sistema. En relación con los procesos escolares, se considera el acceso y trayectoria de los estudiantes en los distintos niveles educativos, la gestión, funcionamiento y organización de los planteles. (Montero-Mora \& CantónCroda, 2020)

La calidad de los servicios debe ser una prioridad para las organizaciones pues se considera un importante antecedente de la satisfacción de los clientes lo cual incide directamente en la fidelización de estos y por tanto en la imagen y posicionamiento en el mercado de las organizaciones. Esto implica que las instituciones de educación se deben 
esforzar por mejorar la calidad académica y los servicios que ofrecen a sus estudiantes, y por medio de esto lograr la acreditación de la calidad por parte de los organismos oficiales, que consideran dentro de los requisitos de certificación, la percepción de los estudiantes sobre ciertos elementos de la calidad de la educación, tales como la gestión institucional, la investigación, la infraestructura, la vinculación con el medio, los servicios online, la responsabilidad social y la docencia. (Flores, et al. 2019).

De acuerdo a (González-Peiteado \& Pino-Juste, 2017), medir la satisfacción del estudiante tiene sentido siempre que vaya acompañada de acciones que promuevan la mejora y la innovación; es por ello que medir dicho constructo de una manera consistente, permanente y adecuada, orientaría a la toma de decisiones correctas intentando aprovechar las oportunidades de mejora.

\section{Medición de la calidad de los servicios educativos}

Muchos son los modelos de calidad del servicio utilizados en la educación, dentro de este contexto es importante analizar cómo se implementan estándares de sistemas de gestión de la calidad en la educación superior con el fin de mejorar la Calidad en los servicios de forma sistémica e integra. (Fontalvo et al. 2020). Otros autores, han utilizado modelos de gestión para mejorar la docencia de manera integral, (Llanos \& Martínez, 2018).

Por su parte (Suárez-Parra \& Zipaquirá-Vargas, 2016) cuantificar la calidad del servicio no es tarea sencilla, se trata de un concepto comportamental con características de intangibilidad, inseparabilidad, heterogeneidad y con intervención del cliente en el proceso.

De acuerdo con lo anterior, medir la satisfacción de los usuarios del servicio "formación educativa" resulta relevante para conocer su conformidad con la calidad del servicio entregado. En este sentido, toda vez que la enseñanza universitaria es un servicio cuyos usuarios son los alumnos, parece evidente que la calidad del servicio puede también establecerse a partir del registro de la satisfacción de éstos en relación con determinados aspectos tangibles e intangibles del mismo. (Palominos-Belmar, et al. 2016)

En la revisión bibliográfica se encontró 3 modelos para medir la calidad de los servicios educativos y éstas son:

El modelo SERVQUAL (Parasuraman et al. 1985) es un método de investigación que goza de reconocimiento y por ende es ampliamente utilizado para evaluar la calidad de 
los servicios tanto en el campo empresarial como en el académico. El modelo lo integran cinco dimensiones: (1) Capacidad de respuesta: disposición del personal para prestar ayuda y servicio rápido a los usuarios. (2) Fiabilidad: definida como la prestación del servicio prometido de modo adecuado y estable en el tiempo. (3) Empatía: capacidad para entender la perspectiva del usuario. (4) Seguridad: atención y habilidades expuestos por los empleados para inspirar confianza y credibilidad. (5) Elementos tangibles: representa la apariencia de las instalaciones físicas, equipos, personal y materiales de comunicación. Consta de (22 ítems)

Por su parte, (Cronin Jr. \& Taylor, 1992), con el modelo SERVPERF que se compone de los 22 ítems de la escala SERVQUAL, pero mide exclusivamente las percepciones de la calidad del servicio, son considerados como los trabajos pioneros en la medición de la calidad del servicio y satisfacción del cliente y han sido aplicados en un gran número de empresas de servicios. Sin embargo, se encontraron investigaciones que realizaron adaptaciones a los modelos SERVQUAL y SERVPERF para medir la percepción de la calidad de los servicios de estudiantes en universidades privadas; e investigaciones en las que se diseñaron los instrumentos también en instituciones de educación superior. (Montero-Mora \& Cantón-Croda, 2020)

El tercer modelo no tan comercial es: SERVQUALing, es un instrumento comportamental de cinco dimensiones tipo Likert y desarrollado a partir del modelo SERVQUAL. Mientras el modelo SERVQUAL plantea una escala compuesta de 22 ítems para medir percepción y 22 ítems para medir expectativas, distribuidos en cinco dimensiones: tangibilidad, fiabilidad, capacidad de respuesta, seguridad y empatía; SERVQUALing conserva los 22 ítems y las cinco dimensiones, con la diferencia que no tiene en cuenta las expectativas, sino que lo hace solo desde la percepción que tienen los clientes respecto al servicio recibido. La propuesta de SERVQUALing afirman que la medición de la calidad del servicio se debe hacer únicamente con base en las percepciones y no en la diferencia entre las percepciones y las expectativas. En su aplicación, SERQUALing ha resultado ser un modelo exitoso en diferentes contextos, entre ellos, el campo educativo. (Suárez-Parra \& Zipaquirá-Vargas, 2016)

\section{ESTRATEGIAS METODOLÓGICAS O MATERIALES Y MÉTODOS}

El procedimiento seguido para realizar esta investigación se describe ahora en detalle. 


\subsection{El diseño y el sitio del estudio}

Éste artículo trata de una exploración sistemática, desarrollado por profesionales doctorandos en Gestión Pública y Gobernabilidad, de la Escuela de Posgrado de la Universidad César Vallejo, Perú. La obtención de datos fue realizada en cuatro meses. Para realizar esta revisión, sobre el análisis de la calidad de los servicios educativos en Latinoamérica, se tomaron artículos científicos de los distintos países cuya lengua oficial es el español o el portugués (Argentina, Bolivia, Brasil, Chile, Colombia, Costa Rica, Cuba, Ecuador, El Salvador, Guatemala, Honduras, México, Nicaragua, Panamá, Paraguay, Perú, Puerto Rico, República Dominicana, Uruguay y Venezuela)

Esta fue una revisión sistemática de alcance desarrollada por alumnos investigadores del doctorado en Gestión Pública y Gobernabilidad de Escuela de Posgrado de la Universidad César Vallejo, en Perú. La obtención de datos fue realizada en noventa días (del 05 de enero al 10 de abril del 2021).

\subsection{Criterios de elegibilidad}

Tipos de intervinientes: Incluye investigaciones que involucran a estudiantes de la educación básica, estudiantes de educación superior en la comunidad en general y literatura teórica. Tipos de estudios: Considerando una gran cantidad de investigaciones sobre la calidad de los servicios educativos, el propósito de esta breve revisión es trazar el conocimiento existente sobre el tema y determinar el modelo de medición de la calidad de los servicios educativos. Tipos de resultados: Se eligieron como resultados primarios a: publicaciones originales de estudios científicos e información reciente. Búsqueda de estudios: Los principales resultados son: publicaciones originales y la información más reciente para la investigación científica. Búsqueda de investigación: utilizamos las referencias de investigación primarias y secundarias que se encuentran en la búsqueda electrónica para búsquedas manuales. La búsqueda se realizó directamente en las bases de datos EBSCO, Scopus, Science Direct, SciELO, ProQuest y Redalyc, utilizando el filtrado de arbitrajes y publicaciones académicas de 2016 a 2021. Selección de la investigación: el proceso de selección de la investigación lo llevan a cabo dos examinadores independientes y un tercer examinador resuelve las diferencias. La selección de artículos científicos se divide en dos etapas. El primer paso es mirar los títulos y resúmenes de los artículos encontrados usando nuestra estrategia de búsqueda; 
seleccionar estudios que cumplan con los criterios. El segundo paso es revisar la redacción completa del artículo preseleccionado para confirmar su elegibilidad.

\subsection{Análisis de datos}

La estrategia de indagación bibliográfica produjo 950 bibliografía. En este proceso de selección, se eliminaron 919 referencias que no cumplían con los criterios de inclusión luego de verificar el título y el resumen. Se leyeron 31 citas seleccionadas para verificar la selección. Una vez completado el proceso de aceptación, se incluirán veintisiete (31) artículos científicos. Los resultados de los 31 artículos restantes se presentan de forma sistemática y descriptiva porque la naturaleza de estos estudios no permite realizar ningún otro tipo de análisis de la misma forma.

\section{RESULTADOS Y DISCUSIÓN}

Se realizó una búsqueda manual utilizando las referencias de los estudios primarios y secundarios encontrados en la búsqueda electrónica. Las búsquedas se realizaron directamente en la base de datos EBSCO, Scopus, Science Direct, SciELO, ProQuest y Redalyc los últimos 5 años.

\section{Las dimensiones de la calidad del servicio educativo con mayor desempeño fueron:}

Servicio documental, la prestación del servicio del centro de atención documental presenta un buen desempeño. (Delahoz-dominguez et al. 2020); además las dimensiones redacción y estructura del comunicado, así como también la dimensión estandarización del proceso de servicio, presentan los valores de sumas de cuadrado más grandes, evidenciando de esta manera que son las dimensiones con el mejor desempeño en la prestación de servicio de la organización.

En el trabajo de (Alaña et al. 2018), se encontró que las necesidades de los usuarios son cubiertas satisfactoriamente, las mismas que se ubicaron en la categoría de muy alta frecuencia, en vista de que los empleados tienen buen desempeño al momento de prestar el servicio, siendo eficiente en el cumplimiento de las actividades laborales, puesto que las condiciones laborales influyen en la productividad de los mismos, para el indicador cumplimiento del servicio se observó que se ofrece comodidad en la prestación del servicio a los usuarios, se establecen acciones recurrentes orientadas a la optimización del servicio. 
Continuando con los resultados de (Alaña et al. 2018), se determinó que se cuenta con los empleados suficientes para ofrecer un servicio eficiente, los mismos cumplen con el servicio ofrecido a sus usuarios, satisfaciendo sus necesidades. Éstos resultados van asociados con (Atia, et al. 2018) donde los resultados muestran cómo las necesidades y expectativas de los clientes difieren en algunos momentos de verdad propiciados en la prestación del servicio, es el caso de los horarios, tiempo de respuesta, trámites, entre otros. Aspectos que al final se convierten en una experiencia personal percibida positiva o negativamente por el cliente.

De igual forma se verificó que se manejan horarios para ofrecer el servicio requerido a sus usuarios, los mismos no permiten satisfacer las necesidades de los usuarios, por lo que se deben ajustar los horarios de atención. También se evidenció que se satisfacen los requerimientos de los usuarios oportunamente, mediante respuestas efectivas y oportunas al momento de solicitar cualquier información. (Alaña et al., 2018)

Además, cabe resaltar el estudio realizado por (Alvarado Lagunas, et al. 2016), afirma que se puede argumentar que las variables con más impacto — de mayor a menor grado- sobre la percepción de la calidad educativa en esta muestra global estriban en medios de enseñanza (41.9\%), desarrollo integral (37.1\%), componente físico $(30.4 \%)$ y planta docente $(23.9 \%)$ ésta última dimensión se relaciona con (Martínez-Luis et al., 2020) donde los servicios relacionados con las funciones docentes son mejor evaluados. Éstos hallazgos concuerdan con el estudio realizado por (Núñez, et al. 2019) en su estudio, señala que el $71,3 \%$ de las profesoras de inicial percibían que en las directoras de las Instituciones Educativas de nivel Inicial del distrito habían desarrollado las competencias administrativas y que el $85 \%$ de las profesoras de inicial perciben que el servicio que brindan en su escuela es de alta calidad y para el $12,5 \%$ el servicio es de calidad.

El estudio realizado por (Alcas Zapata et al. 2019) afirma que el 25,3\% de los docentes perciben que la calidad de servicio presenta un nivel regular, el 44,2\% de los docentes perciben la calidad de servicio en el nivel buena y un 30,5\% de los docentes perciben la calidad de servicio en el nivel muy buena. Éstos hallazgos van alineados a lo referido por (Vergara Morales, et al. 2018), (1) el interés por los contenidos curriculares se asoció con el gusto por lo aprendido, (2) la satisfacción con la decisión de cursar la asignatura se asoció tanto con el gusto por lo aprendido como con el entusiasmo por los contenidos transmitidos y (3) el disfrute de las clases se asoció con la estimulación en la enseñanza. 
Se pudo constatar que los buenos resultados mediante la aplicación de las fases: planificación, configuración-búsqueda de dispositivos, autorización previa, envío del mensaje, interacción del contenido y evaluación. Permite que estudiantes y sociedad en general cuenten con información oportuna de los productos y/o servicios académicos ofrecidos por las instituciones de educación superior, (Medina Chicaiza, et al. 2019). Éstos hallazgos se relacionan con (González-Peiteado \& Pino-Juste, 2017), donde la satisfacción estudiantil como la apreciación favorable que hacen los estudiantes de los resultados y experiencias asociadas con su educación, en función de la atención a sus propias necesidades y al logro de sus expectativas.

Se encontró además estudios que difieren a los resultados anteriores, tal es el caso de (Palacios et al., 2020) el $82.9 \%$ de los usuarios tienen una percepción regular a mala acerca de la calidad del servicio educativo; éstos resultados se alinean con (Zambrano R, et al. 2019) que al clasificar los factores: ambiente educativo, bienestar estudiantil, calidad administrativa, calidad docente, infraestructura educativa, organización académica, recursos para la investigación, servicios estudiantiles, servicios informáticos y vinculación con la sociedad. Al comparar las puntuaciones de importancia y satisfacción, se encontró que los estudiantes están insatisfechos con todos los factores.

En los dominios de la calidad de los servicios educativos, hubo una brecha negativa entre las expectativas de los estudiantes y las percepciones de los servicios educativos ofrecidos, esto significa que la calidad de los servicios entregados fue menor a la esperada por los estudiantes, (Basantes, et al. 2019), éstos resultados se asemejan al estudio realizado por (Martínez-Luis et al., 2020) los servicios relacionados con los sistemas de inscripción y servicios al estudiante reciben calificaciones bajas, lo cual refleja la poca atención que reciben los estudiantes por parte del personal que labora en estas áreas.

Por último en los resultados obtenidos en (Díaz, 2018) que si bien es cierto que la dimensión ha obtenido el 43\% entre los valores "Bajo" y "Muy Bajo", es decir, por debajo del 50\%, encontramos que "Medio" es el siguiente valor con mayor porcentaje, es decir, la mayoría de los alumnos no perciben una mala calidad en esta dimensión, pero tampoco la perciben con una alta calidad, esto se debe a que aunque por un lado se han realizado mejoras significativas en la infraestructura y en el mobiliario, por otro lado, es contrarrestado con servicios negativos como, por ejemplo, la mala atención que se tiene en la caja. Por otro lado, tanto en la encuesta a docentes como en la encuesta al personal 
administrativo, los valores "Bajo" y Muy Bajo" superan el 50\%, a ello se añade el hecho de que en las entrevistas al personal directivo y administrativo se obtuvo como resultado el valor "Muy Bajo" con un 100\%, esto se debe básicamente a que la institución no cuenta con un sistema de intranet tal como lo solicitan los estándares de evaluación.

\section{CONCLUSIÓN O CONSIDERACIONES FINALES}

Con base en los resultados de calidad de servicio obtenidos en las instituciones educativas de nivel superior, se puede inferir que los usuarios de estas entidades tienen expectativas sobre los servicios que necesitan. Es por esto que estas instituciones no solo deben enfocarse en los productos y servicios que brindan, también deben cumplir con las condiciones para que los usuarios (en este caso, estudiantes) reciban los servicios. Por tanto, la importancia de estas entidades radica en determinar las necesidades de sus clientes con el fin de establecer un servicio de apoyo logístico en base a estas necesidades, y el servicio logístico debe estar $100 \%$ orientado al cliente.

Basándose en los artículos estudiados, se puede concluir que los estudiantes perciben como Bueno y Muy Bueno, el nivel de la calidad global de los servicios educativos en Latinoamérica por lo que la mayoría de las dimensiones de calidad inherentes al servicio educativo estuvieron por encima del nivel esperado y que su rendimiento fue bueno, ya que mejoran de un periodo a otro.

Así mismo, se pone de manifiesto la preponderancia del modelo SERVQUAL para medir la calidad, ya sea aplicando las mismas técnicas de investigación del modelo o aplicando otras para confirmar los atributos de la calidad del servicio.

\section{LISTA DE REFERENCIAS}

Alaña, J., Rosales, C., \& Romero, F. (2018). Calidad de servicio en los Consejos de Desarrollo Científicos y Humanísticos de las Universidades Públicas. In Crescendo, 9(3), 435-456. Retrieved from https://revistas.uladech.edu.pe/index.php/increscendo/article/view/1959

Alcas Zapata, N., Alarcón Díaz, H. H., Venturo Orbegoso, C. O., Alarcón Díaz, M. A., Fuentes Esparrell, J. A., \& López Echevarria, T. I. (2019). Tecnoestrés docente y percepción de la calidad de servicio en una universidad privada de Lima. Propósitos $\quad y \quad$ Representaciones, $\quad 7(3), \quad$ 231-247. https://doi.org/http://dx.doi.org/10.20511/pyr2019.v7n3.388

Alonso Dos Santos, M. (2016). Calidad y satisfacción : el caso de la Universidad de Jaén. 
Revista de La Educación Superior, 45(178), 79-95. https://doi.org/http://dx.doi.org/10.1016/j.resu.2016.02.005

Alvarado Lagunas, E., Morales Ramírez, D., \& Aguayo Téllez, E. (2016). Percepción de la calidad educativa : caso aplicado a estudiantes de la Universidad Autónoma de Nuevo León y del Instituto Tecnológico de Estudios Superiores de Monterrey. Revista de La Educación Superior, 45(180), 55-74. https://doi.org/10.1016/j.resu.2016.06.006

Araya-castillo, L., Escobar-farfán, M., Bertoló, E., \& Barrientos, N. (2016). Propuesta de modelo para calidad de servicio en escuelas de negocios en Chile. Pensamiento y Gestión, $91-115 . \quad$ Retrieved from https://rcientificas.uninorte.edu.co/index.php/pensamiento/article/view/8675/214 421442353

Arciniegas, J., \& Mejías, A. (2017). Percepción de la calidad de los servicios prestados por la universidad militar nueva granada con base en la escala servqualing, con análisis factorial y análisis de regresión múltiple. COMUNI@CCIÓN, 8(1). Retrieved from http://www.scielo.org.pe/scielo.php?script=sci_arttext\&pid=S221971682017000100003

Atia, V., Stefanell, Í., Miranda, P., \& Salgado, R. (2018). Calidad de servicio en una institución de educación superior en la ciudad de Barranquilla. Ciencias Administrativas, (11), 27-40. Retrieved from https://www.redalyc.org/articulo.oa?id=511654337003

Basantes, R., Vinueza, A., Coronel, J., Davalos, E., \& Miño, G. (2019). Brecha según el modelo servqual entre las expectativas y las percepciones de los estudiantes con respecto a los servicios educativos en la Universidad Nacional de Chimborazo. Didasc@lia: Didáctica y Educación, X(1), 135-146. Retrieved from https://dialnet.unirioja.es/servlet/articulo?codigo=7242006

Contrera, E., Fraile, A., \& Suárez, A. (2019). Análisis de la calidad de los servicios académico- administrativos en una universidad colombiana. Revista Lasallista de Investigación, $16(1)$, 47-61. https://doi.org/http://dx.doi.org/10.22507/rli.v16n1a9

Cronin Jr., J. J., \& Taylor, S. (1992). Measuring Service Quality - A Reexamination And 
Extension. Journal of Marketing, 56(3), 55-68. https://doi.org/10.2307/1252296

Delahoz-dominguez, E. J., Fontalvo, T. J., \& Fontalvo, O. M. (2020). Evaluación de la calidad del servicio por medio de seis sigma en un centro de atención documental en una universidad. Formación Universitaria, 13(2), 93-102. Retrieved from http://dx.doi.org/10.4067/S0718-50062020000200093

Díaz, V. (2018). Calidad del servicio educativo utilizando los estándares del SINEACE, el caso de la carrera administración de negocios internacionales de un ISTP. CONSENSUS, 21(1), $59-70$. https://doi.org/https://doi.org/10.33539/consensus.v21n1.983

Flores, G., Flores, D., \& Romero, A. (2019). Propuesta de instrumento para evaluar la calidad de los servicios y satisfacción de los usuarios en el proceso de inscripción de una institución de educación superior. Revista Dilemas Contemporáneos: Educación, Política y Valores, VI(2), 1-19. Retrieved from https://www.dilemascontemporaneoseducacionpoliticayvalores.com/index.php/d ilemas/article/view/508

Fontalvo, O. M., Fontalvo, T. J., \& Herrera, R. (2020). Monitoreo y control del rendimiento de las dimensiones de calidad de un centro de atención del servicio en una institución de educación superior. Información Tecnológica, 31(3), 113121. https://doi.org/http://dx.doi.org/10.4067/S0718-07642020000300113

González-Peiteado, M., \& Pino-Juste, M. (2017). Estudio de la satisfacción percibida por los estudiantes de la UNED con su vida universitaria. Revista Iberoamericana de Educación a Distancia, 20(1), 243-260. https://doi.org/http://dx.doi.org/10.5944/ried.20.1.16377

Llanos, M., \& Martínez, A. A. (2018). Diseño e Implementación de un Modelo de Gestión en Docencia Universitaria : Caso Carreras de Ingeniería de la Universidad Arturo Prat, Chile. Formación Universitaria, 11(6), 3-16. https://doi.org/http://dx.doi.org/10.4067/S0718-50062018000600003

Martínez-Luis, D., Pérez-Fernández, A., Pat Fernández, L., \& García Rodríguez, J. (2020). La importancia de la calidad en la universidad pública . La percepción del estudiante en la Universidad Autónoma del Carmen. Revista Iberoamericana Para La Investigación y El Desarrollo Educativo, 10(20). https://doi.org/https://doi.org/10.23913/ride.v10i20.658 
Medina Chicaiza, R., García García, J., \& Jarrín Arias, J. (2019). Marketing de proximidad: una herramienta de promoción de productos o servicios educativos. $3 C$ Empresa. Investigación y Pensamiento Crítico, 8(2), 44-67. https://doi.org/http://dx.doi.org/10.17993/3cemp.2019.080238.44-67

Montero-Mora, J. G., \& Cantón-Croda, R. M. (2020). Validación de un instrumento para medir satisfacción de usuarios en instituciones educativas del sector privado: ciudad de Xalapa-Enríquez ( México ). Revista Innovaciones Educativas, 22(32), 122-136. https://doi.org/https://doi.org/10.22458/ie.v22i32.2727

Núñez, L., Menacho, I., Giordano, M., Uribe, Y., \& Rodriguez, L. (2019). Competencias administrativas en la calidad del servicio en las instituciones educativas del nivel inicial. Revista de Investigación Apuntes Universitarios, 9(1), 19-36. Retrieved from http://repositorio.usil.edu.pe/handle/USIL/8671

Palacios, F., Damián, E., \& Damián, N. (2020). Relación entre el clima organizacional y la calidad de servicio en una institución educativa del Distrito Santa Anita , Perú , Año 2017. Revista Científica y Tecnológica UPSE, 7(1), 1-7. https://doi.org/https://doi.org/10.26423/rctu.v7i1.502

Palominos-Belmar, P.-I., Quezada-Llanca, L.-E., Osorio-Rubio, C.-A., \& Torres-Ortega, Jorge-Antonio Lippi-Valenzuela, L.-M. (2016). Calidad de los servicios educativos según los estudiantes de una universidad pública en Chile. Revista Iberoamericana de Educación Superior, vii(18), 130-142. https://doi.org/https://doi.org/10.22201/iisue.20072872e.2016.18.180

Parasuraman, A., Zeithaml, V. A., \& Berry, L. L. (1985). A Conceptual Model of Service Quality and its Implication for Future Research (SERVQUAL). Journal of Marketing, 49(4), $41-50$. https://doi.org/https://doi.org/10.1177/002224298504900403

Regina Ferreira, A., \& De la Cruz, A. (2019). La calidad de los servicios educativos y la satisfacción del estudiante extranjero de la Universidad Peruana Unión. Revista de Investigación Valor Agregado, 6(1), 9-26. Retrieved from https://revistas.upeu.edu.pe/index.php/ri_va/article/view/1285

Sotelo, J., \& Figueroa, E. (2017). El clima organizacional y su correlación con la calidad en el servicio en una institución de educación de nivel medio superior. Revista Iberoamericana Para La Investigación y El Desarrollo Educativo, 8(15), 582- 
609. https://doi.org/https://doi.org/10.23913/ride.v8i15.312

Suárez-Parra, A. B., \& Zipaquirá-Vargas, A. A. (2016). Validación de la escala SERVQUALing en la población de estudiantes de ingeniería de la Universidad de Boyacá . Caso servicios bibliográficos. Educación En Ingeniería, 11(22), 24-30. https://doi.org/https://doi.org/10.26507/rei.v11n22.639

Turpo, J., \& Jaimes, D. (2017). Estudio de los vínculos entre cultura organizacional y calidad de los servicios educativos en la imagen institucional de una universidad privada confesional. Apuntes Universitarios. Revista de Investigación, 7(1), 97116. https://doi.org/https://doi.org/10.17162/au.v7i1.203

Vergara Morales, J., Del Valle, M., Díaz, A., \& Victoria Pérez, M. (2018). Adaptación de la Escala de Satisfacción Académica en Estudiantes Universitarios Chilenos. Psicología Educativa, 24(2), 99-106. https://doi.org/https://doi.org/10.5093/psed2018a15

Zambrano R, J., Loachamín Marcillo, M., Pilco Gallegos, M., \& Pilco Gallegos, W. J. (2019). Cuestionario para medir la importancia y satisfacción de los servicios universitarios desde la perspectiva estudiantil. Revista Ciencia Unemi, 12(30), $35-45$. 7737vol12iss30.2019pp35-45p 